\title{
PROPERTIES OF RADIOACTIVE SMALL IONS AND SIZE DISTRIBUTION OF RADIOACTIVE AEROSOLS IN TOKYO
}

\author{
S.Nakae,J.Y.Moon ${ }^{+}$, T.Yamada, K.Hara and K.Miura \\ Department of Physics, Science University of Tokyo \\ Kagurazaka,Shinjukuku,Tokyo 162-8601,Japan. \\ + Korea Institute of Nuclear Safety \\ Yusung, Daejon City 305-600, Korea.
}

\begin{abstract}
Properties of radioactive ions are examined by using a mobility separation chamber. These small ions are attached to aerosol particles and form radioactive aerosols. The size distribution of natural aerosols are measured with a differential mobility analyzer(DMA) and a light scattering particle counter during the year. Thus the size distribution of radioactive aerosols is calculated from the combination coefficients of ions to aerosols and the measured size distributions of natural aerosols.

As a result, bimodal distribution with peaks of about 0.4 and 1.5 $\mathrm{cm}^{2} / \mathrm{sec}$.volt were found on the radioactive ions in room air. By using the value of $0.4 \mathrm{~cm}^{2} / \mathrm{sec}$.volt of cluster ions, the peak of size distribution of radioactive aerosols was found on about $0.04 \mu \mathrm{m}$ in radius and shifted toward larger size region as compared with those of natural aerosols.
\end{abstract}

Key words: radioactive ions, radioactive aerosols, electrical mobility.

\section{Introduction}

The physical properties and concentration of the radioactive substances in the air are very important from the viwpoint of atmospheric physics and health physics. The presence of these substances influences the ionization of the atmosphere(controls air conductivity) and the lung dose of humans.

Radon in the atmosphere emanates from soil and rocks, and is also releases from coalfired power plants. The radioactive decay products of radon are found in the form of small positive ions in the air. The mobility spectrum of these radioactive ions has been determined by the utilization of a Zeleny tube[Blanc et al., 1963] and an Erikson spectrometer [Bricard, et al., 1966., Fontan, et al., 1969.,Kulju, et al., 1987], and the alpha-activity of equal length segments of collecting electrode has been measured by various devices, such as $\mathrm{ZnS}$ scintillators, nuclear emulsions and surface barrier detectors. However,it is difficult to know the properties of ions under various conditions of atmosphere because of the time resolution of devices. In this paper, the instrument is developed to determine the electrical mobilities of radio-active small ions continuously and the size distributions of radioactive aerosols can be obtained successfully by the use of these results.

\section{Experiment}

(a.) Radioactive small ions.

The mobility spectrometer used here is basically the condenser of parallel plate type. The radioactive ions are introduced into the condenser along the surface of one electrode made by Al-plate. The inside face of 


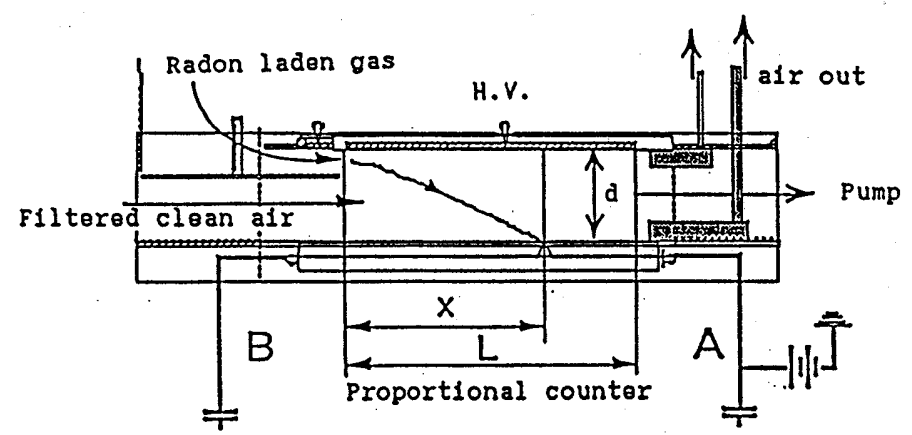

Fig.1. Schematic diagram of the mobility spectrometer of parallel plate type. In the outlet, the flowrate of air-out channel is adjusted to the same with that of Radon laden gas, and keep air in laminar. Pumping air is filtered and returned to inlet clean air.

another plate, on which the ions are deposited, is covered with a thin gold-plated myler film and serves as the window of a proportional gas flow counter. The mobility spectrometer was modified to provide a uniform air flow from two channels at the entrance, one for the filtered radon free air and the other for the radon laden atmosphere. The modified spectrometer is schematically shown in Figure 1. The electric potential is also applied between the plates. The vertical electric field $E$ is provided through the spectrometer so that the radioactive ions are drawn toward the counter surface and deposited at the distance $x$ from the entrance according to their electrical mobilities. The alpha-rays emmited from radioactive ions are detected by the counter through the thin film window. With the known distance $d$ between the radon laden gas channel and the detector window plate, the electrical mobility of ions $k$ can then be determined from $k=v d / E x$, where $v$ is gas velocity.

\section{Signal processing}

The counter is constructed using RC position encoding. The charge division method determines the position of the event by calculating the ratio of the charge collected at one end of the resistive anode wire to the total collected charge on both ends. Figure 2 shows a block diagram of the electronics used to read the position of alpha decay events. Each end of resistive anode wire is connected to a charge sensitive amplifier respectively, and is used as signal output terminals.

The unipolar outputs of the amplifiers are fed to a sum and invert amplifier. It has two inputs to permit the summation of signals from separate circuits. The summed and inverted signal is furnished through the output terminal, and the inverted signals of individual input pulses are also given at another

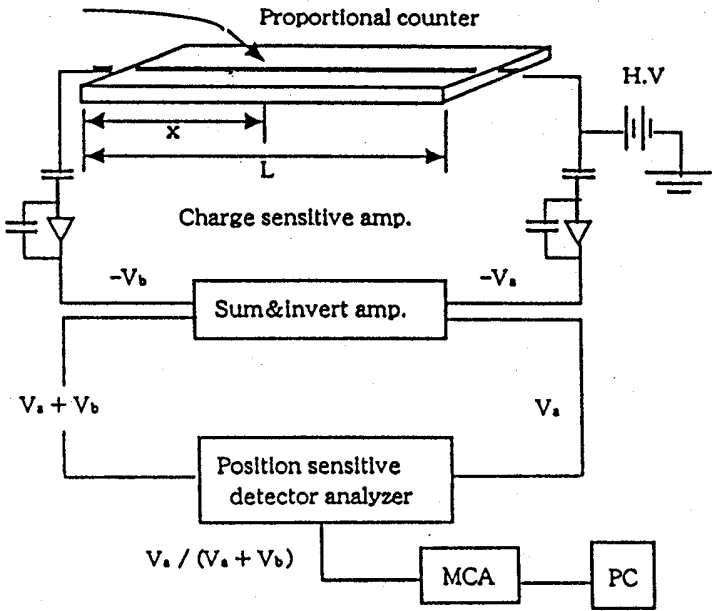

Fig.2. Construction of position sensitive proportional counter. 
output terminals. The amplifier has a rise time of $<50 \mathrm{~ns}$, a bandwidth from $\mathrm{DC}$ to $7.0 \mathrm{MHz}$ and output impedance is $<0.1 \mathrm{ohm}$ for line drive. The output signal is true only when there is a time coincidence of arrival of the input pulses. Position sensitive detector analyzer at the next stage has two input signals. One of the signals has a peak pulse amplitude proportional to the full energy absorbed from an event in the detector. The other signal has a peak pulse amplitude that is a significant fraction of that for the full energy pulse, and the fractional ratio indicates the relative point of impingement of the event along the length of the detector. Thus the ratios of oneside output pulses to the sum of both ends pulses are proportional to the position of generating pulses. These pulse ratios can be observed by the use of a multichannel pulse height analyzer.

(b) Size distribution of natural aerosols

For particles larger than $0.1 \mu \mathrm{m}$ in diameter, the particle size distribution is measured by analysing the light scattered by each particle. Size distributions are obtained by using the optical particle counter of commercial type(RION $\mathrm{KC}-18$ ). Particle concentration is diluted $1: 100$ with clean air and count number of particles are corrected for coincidence errors. For smaller sizes, the measurements of electrical mobilities of particles are most often utilized. Mobility analyzer used in this study consists essentially of coaxial cylindrical condenser with two flow inlets and two outlets, and the inner electrode is divided into two portions, first and second sections. The diameter of inner electrode is $6 \mathrm{~cm}$ and that of outer electrode is $7 \mathrm{~cm}$. The lengths of the first and second section of inner electrode are 18 and $11.5 \mathrm{~cm}$ respectively. The clean air, in which the particles and ions are filtered out by using absolute filter and electrical filter, enters the analyzer through an axial pipe. The air stream is kept laminar during the experiments. The particles are introduced into the analyzer in the form of annular ring around a core of clean air. If the voltage applied to the outer electrode is constant, the particles are classified along the inner collecting electrode according to their electrical mobilities. The ionic current to the second section of inner electrode is measured by a vibrating reed electrometer. By varying the voltage in a systematic manner, the mobility distribution of particles can be obtained from the current of second section of inner electrodes. The voltage steps are chosen such that each mobility region would form adjoining and nonoverlapping intervals.

\section{Conversion of mobility distribution to size distribution}

Particle radii are derived from mobilities using the Stokes-CunninghamMillikan formula, and the stationary charge distribution on the particles in a symmetrical bipolar ionic atmosphere was determined theoretically by Fuchs[1963]. However the difficulties in converting a mobility distribution to a size distribution arise because a large particles carrying a multiple number of electronic charges cannot be distinguished from smaller particles carrying a single electronic charge. The correction of this is derived by a slight modification of Hoppel method[Hoppel,1978]. The first approximation assumes that all charged particles are singly charged particles, and the first order size distribution is then obtained with the relationship between the total number of particles and the number with charged particles at every size interval. For the second approximation, singly and multiply charged particles are estimated using the first ap- 
proximation. New singly charged particles are obtained by subtracting the multiply charged particles and the new estimate of singly charged particles is then used to calculate the second approximation to the size distribution. For the higher approximations, this process is repeated using the newly found approximation. Size distributions are obtained successfully with the first three approximations. In our case, the charged fraction of particles obeys the results of Fuchs theory, instead of Boltzmann charge distributions.

\section{Results and Discussions}

\section{(a)Radioactive small ions}

As shown in Figure 2, the detective device is essentially a rectangular gas flow counter. The cathode of counter is a chrome plated iron mesh covered with thin gold-plated mylar film of $0.85 \mathrm{mg} / \mathrm{cm}^{2}$ thick, which allows alpha particles emitted from deposited radioactive ions on the surface to penetrate through it. Carbon wire was used as the anode of the counter. Both ends of carbon wire are connected to charge sensitive preamplifiers respectively. Commercial methane of 99 percent purity is circulated through the counter. The values of alpha plateau length and slope of the counter were found to be 250 volts and few percent per 100 volts. The source employed in this measurement was a radium $D+E+F$ source emitting both alpha and beta particles.

The mobility distributions of radioactive small ions can be obtained from the position analysis of detected events. The relation between positions of detected events and observed channels on a pulse height analyzer is shown in Figure 3. In this case, the position of the source is changed in stepwise along the axis of counter. The spatial uncertainty in position is found as $\pm 4 \mathrm{~mm}$.

An alpha particle generally produces an electron-ion track along the trajectory of the particle in the gas flow counter. For a trajectory that is not always perpendicular to the anode of the counter, the concept of an interaction point

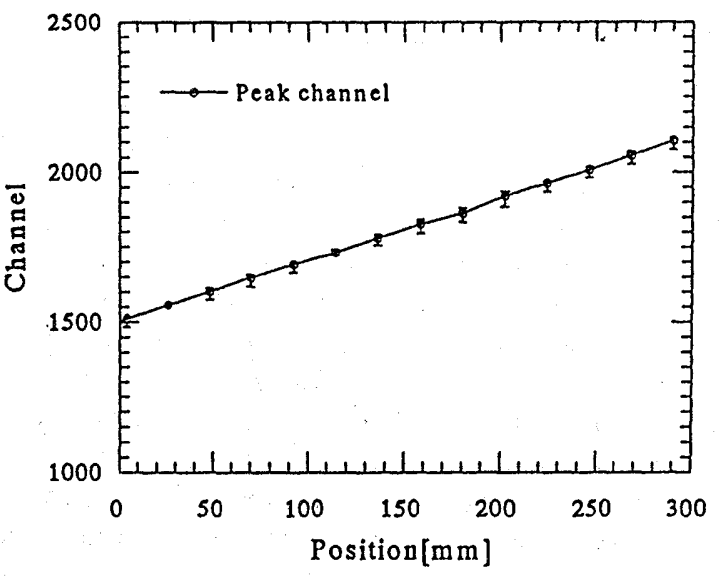

Fig.3. The relation between the positions of detected events and observed channels on the pulse height analyzer. The spatial uncertainty in position is found as $\pm 4 \mathrm{~mm}$.

is not valid, because the interaction is spread over a space interval $\Delta x$ that has a width $w=d \cos \alpha$, where $d$ is the track length inside the counter and $\alpha$ is the track angle with respect to the anode. For such particles, the center of charge position is in $\Delta x$ and depends on the specific ionization of the electron-ion track.

From linear relation between the position of detected events and the observed channels on the pulse height analyzer, the mobility distribution of radioactive small ions can be obtained successfully. In this case, the background alpha pulses, caused by radioactive impurities in the counter materials, appeared roughly in uniformity along the axis of counter. Then the signals can be obtained by deducting the background count from the total amount. As a results, bimodal distributions with peaks of about 0.4 
and $1.5 \mathrm{~cm}^{2} / \mathrm{sec}$.volt are found on the naturally occurring radioactive small ions. They seem to be shifted up to the higher mobility side in the room air. It was found with peaks of $0.4 \sim 0.5$ and $1.5 \sim 2.0 \mathrm{~cm}^{2} / \mathrm{sec}$.volt. respectively. The values of mobility varies in a given atmospheric condition, especially effective in humidity of the air. The record of mobility distributions of radioactive small ions is shown in Figure 4.

The reason of bimodal distribution is not clear exactly, however it seems likely that the one peak in larger mobility range is due to primary multiple charged ions, and the other is secondary single charged ones, namely the cluster ions.
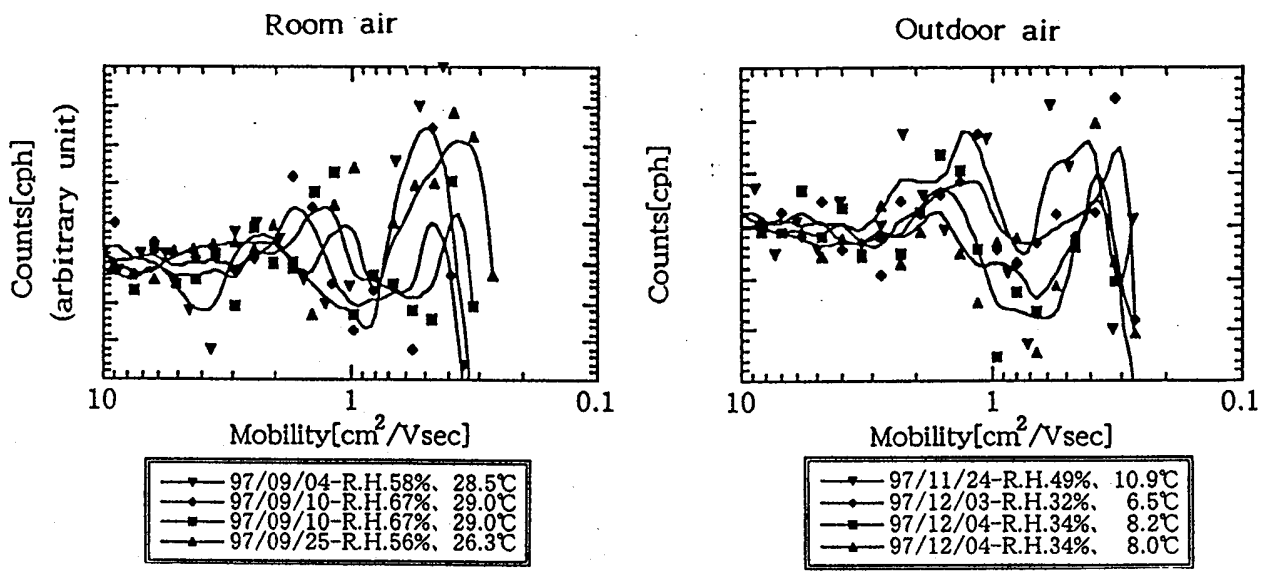

Fig.4. Mobility distributions of radioactive small ions.

(b) Size distribution of radioactive particles

Radioactive small ions, formed by disintegration of radon in the atmosphere, are usually attached almost instantaneously to the extremely numerous natural aerosols[Lassen,1961]. The production rate of radioactive particles of radius $r, A(r)$, is expressed by the following equation

$$
A(r)=\eta_{a}(r) n_{a} N(r)
$$

where $n_{a}$ is the concentration of radioactive small ions, $\eta_{a}(r)$ is the combination coefficient between radioactive small ions and aerosol particles of radius $r$, and $N(r)$ is the total number of natural aerosol particles of radius $r$. Let $L_{0}$ be the neutralised fraction of radioactive small ions and $L_{ \pm 1}$ be a fraction of positive or negative charged ones, and also let $F_{0}$ be a neutralized fraction of aerosol particles, $F_{ \pm p}$ be a fraction of p-positive or p-negative charged particles. Above equation is expressed as,

$$
\begin{aligned}
A(r)= & n_{a} N(r)\left[\eta_{n} L_{o}+\eta_{0}\left(L_{+1}+L_{-1}\right) F_{o}\right. \\
& +L_{+1} \sum_{P=1}^{\infty}\left(\eta_{+p} F_{+p}+\eta_{-p} F_{-p}\right)+L_{-1} \sum_{P=1}^{\infty}\left(\eta_{-p} F_{+p}+\eta_{+p} F_{-p}\right)
\end{aligned}
$$

where $\eta_{0}$ is the combination coefficient between the radioactive small ions and the neutral particles, $\eta_{ \pm p}$ are that of the radioactive ions and 
p-charged particles of the same or opposite signs.

$\eta_{n_{1}}$ is the combination coefficient between neutralized radioactive small ions and aerosol particles. The negatively charged radioactive small ions are not found in the atmosphere. Thus the coefficient will be rewritten in the following form,

$$
\begin{aligned}
\eta_{a}(r)= & L_{o} \eta_{n} F_{o}+L_{+1} \eta_{-1} F_{-1} \\
& +\sum_{P=1}^{\infty}\left(L_{o} \eta_{n} \frac{1}{2} F_{o}+L_{+1} \eta_{+(p-1)} \frac{1}{2} F_{p-1}\right)+\sum_{P=1}^{\infty}\left(L_{o} \eta_{n} \frac{1}{2} F_{o}+L_{+1} \eta_{-(p+1)} \frac{1}{2} F_{p+1}\right)
\end{aligned}
$$

where the fractions are $F_{+p}=F_{-p}=\frac{1}{2} F_{p}$ in symmetrical bipolar ionic atmosphere. These coefficients. $\eta_{n}, \eta_{0}$ and $\eta_{ \pm p}$ for radioactive ions were calculated by Baust[1967] as the functions of $r$. In our case, mobility of radioactive small ions(B) is $0.4 \mathrm{~cm}^{2} / \mathrm{sec}$.volt. and diffusion coefficient (D)is given as $\mathrm{D}=\mathrm{kTB} / \mathrm{e}$, where $\mathrm{k}$ is Boltzmanns constant and $\mathrm{T}$ is the temperature, and $e$ is the elemental charge. As a first approximation, size distribution is given as the combination between positive small ions and neutral particles because of small fractions of neutralized radioactive small ions.

Results are shown in Figure 5. Size distributions of natural aerosols $N(r)$ may be classified roughly into two groups. Type $A$ exceeds in number concentration of nuclei mode particles, and typeB exceeds in that of accumulation mode. They appeared respectively in about 30 and $70 \%$. Although the distribution varies from time to time, there is stableness as a monthly mean in Tokyo. Size distributions of radioactive aerosols $A(r)$ are calculated from that of natural aerosols $\mathrm{N}(\mathrm{r})$ as shown in Fig.5. The distributions of radioactive aerosols are shifted toward the larger size region as compared with those of natural aerosols and in many cases, the peak of $A(r)$ appeared in the size of about $0.04 \mu \mathrm{m}$ in radius. In the case of typeA, the values of peak are shifted down to smaller size regions. This is characterized by an increase of aerosol concentration near the source of particles.
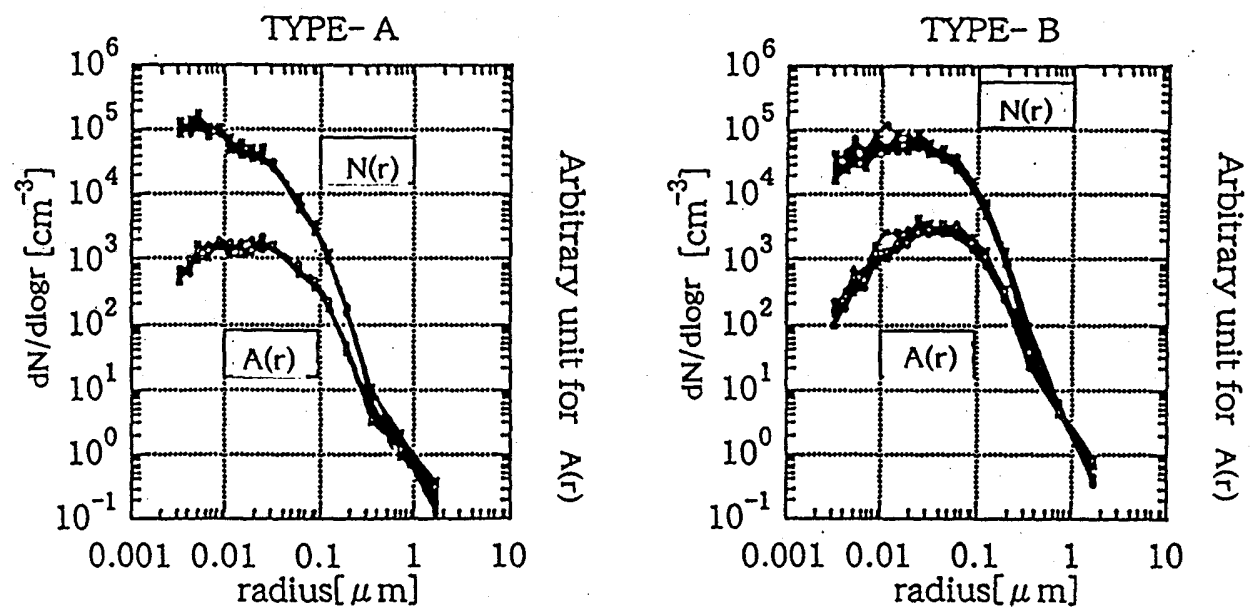

Fig.5. Size distributions of radioactive aerosols[A(r)] in the atmosphere. $N(r)$ is that of natural aerosols. 


\section{Conclusion}

A new method for obtaining the electrical mobilities of radioactive small ions was developed. The apparatus is basically the condenser of pararllel plate type and the electric potential is applied between the plates. The radioactive small ions introduced into the condenser along one electrode are deposited on the plate surface of opposite polarity according to their electrical mobilities. The collecting surface is a thin Au-plated myler film and serves as the window of a position sensitive proportional counter using resistance-capacitance position encoding. Then the activity distribution on the collecting surface along the axis of the counter can be obtained successfully. As a results, bimodal mobility distributions were found with peaks of about 0.4 and $1.5 \mathrm{~cm}^{2} / \mathrm{sec} . v 0 l t$. in the atmosphere.

These radioactive small ions are attached to aerosol particles and formed radioactive aerosols. The size distribution of radioactive aerosols can be calculated from the combination coefficient of ions to aerosols and size distributions of natural aerosols. The peaks of number concentration of the radioactive aerosols were found on about $0.04 \mu \mathrm{m}$ in radius and these humps of distributions are shifted toward larger size region as compared with those of natural aerosols. When the natural aerosols increase in number at nuclei mode, the peak of size distribution of radioactive aerosols shifts down to smaller size region.

\section{References}

Baust,E.,: Die anlagerung von radioaktiven atomen und ionen an aerosolteilchen.,Z.Physik.,199, 187-206,1967.

Blanc,D.,J.Fontan and M.Juan.: On the mobilities of natural ions obtained in filter air. Compte Rendus de I'Academie des Sciences de Paris,260, 2099-2100,1963.

Bricard,J.,F.Billard,D.Blanc,M.Cabane and J.Fontan.: Detailed structure of the mobility spectrum of the small radioactive ions in the air. Compte Rendus de I'Academie des Sciences de Paris, 263,761-764,Series B, 1966.

Fontan,J.,D.Blanc,M.L.Hubertas and A.M.Mart.,: Mesure de la mobilite et der coefficient de diffusion des particules radioactives. Elementary Electrodynamics(S.C.Coronitte and J.Hughes,eds),257-267. Gordon and Breach Science Pub.,New York.1969.

Fuchs,N.A.,: On the stationary charge distribution on aerosol particles in a bipolar ionic atmosphere., Geof.pura e appl.,56,185-193,1963.

Hoppel,W.A.,: Determination of the aerosol size distribution from the mobility distribution of the charged fraction of aerosols., J.Aerosol Sci.,9, 41-54,1978.

Kulju,L.M.,K.D.Chu and P.K.Hopke.,: Development of amobility analyzer for studying the particle producing phenomena related to radon progeny.,

Radon and Its Decay Products(P.K.Hopke,ed.) 357-364,American Chemical Society, Washington,D.C., 1987.

Lassen,L.,: The attachment of natural radioactivity to aerosols.,Geof.pura e appl.,50,281-285,1961.

(Received November 29, 1999; revised December 21, 1999; accepted January 13,2000 ) 